\title{
Posture Recognition and Imitation using Haar Wavelet Transform and Neural Net- works
}

\author{
J. Yaser Daanial Khan \\ School of Science and Technology \\ University of Management and Technology \\ Lahore 54000, Pakistan \\ yaser.khan@umt.edu.pk \\ M. Khalid Mahmood \\ Department of Mathematics \\ University of the Punjab \\ Lahore 54000, Pakistan \\ khalid.math@pu.edu.pk
}

\begin{abstract}
Human postures or gestures which essentially are static orientations of the body usually symbolizing a motive are put to use for bridging the gap between novice and expert users. Both the users are at the same natural level and capability to interact. To achieve an interaction between the machine and its human operator various techniques are suggested in the text. Their implementations though not cheap are also available in the market. Our research and implementation revolves around the use of an ordinary inexpensive camera to acquire live video and hence segment the human forearm locations using various filters. A database containing nearly 2000 images was created for this purpose. Furthermore a neural network for the purpose of pattern recognition among segmented images was trained. All the experimental results are gathered through testing on a live application in an interactive scenario.
\end{abstract}

Keywords: Neural Networks, Posture Recognition, Human Computer Interaction

Received: 2 May 2018, Revised 6 June 2018, Accepted 17 June 2018

DOI: $10.6025 / \mathrm{jic} / 2018 / 9 / 4 / 133-143$

(C) 2018 DLINE. All Rights Reserved

\section{Introduction}

The human posture recognition systemsare used in various current state of art applications. One function, which shall be the focus of this article, is the use of recognized postures and their subtle application. The recognized motions or actions are mapped on to a model to make it mimic same postures. A vital area of interest in this research work is to segment human body from background, indentify different limbs and follow their movement. A human like model is simulated in a $3 \mathrm{D}$ virtual environment which imitates those postures. Each of the essential aspect regarding this application is discussed such as method of extraction of human body from a stream of images, removal of background, reduction of noise from the image, division of human body according to fixed proportion of each part, finding the exact positioning of each part of the body and invocation ofoperationsaccording to the gestures. Initiallyin section 2. The current state of art systems is discussed.In section 3 a brief 
description of the system is elaborated. Section 4. explains in detail the "system model", this phase majorly covers the preprocessing operations. Section 5.explains the structure of our ArtificialNeural Network along with its parameters. Section 6. is the esting phase of the proposed system. Section 7 and 8 deals with the character modeling and its animation in Microsoft XNA frameworks. Last but not the least, some results and conclusions are provided.

\section{Existing Research and Systems}

Due to considerable work that has been already done in the fields relating to the proposed problem abundant text is available. In [1] the author proposes a gesture recognition method based on local motion learning.. They have used Histogram of Oriented Gradients (HOG) as descriptor to reach perfection in tracking. For posture recognition, the classification was done through knearest neighbor rule. Another article on intelligent human body tracking, modeling, and activity analysis of video surveillance system by K. Srinivasan et. al. [2] refers to tracking feature points of a human body to predict the motion in the next frame of the video. A well established model for posture detection is Adaptive Neuro-Fuzzy Interference System (ANFUS) model. [3] This model is used for recognition of sign languages. They used a colored glove to vacillate image segmentation. The use of colored glove helped in deducting the hand gesture significantly. Another effective method is named Adaptive Self-Organizing Color Segmentation Algorithm fabricated from the work of Ying Wu et. al. [4] This method uses the ASCS (adaptive self-organizing color segmentation) algorithm and a transductive learning algorithm to find the position of the human forearm or hands. Some related work has been established by Bernard Boulayet. al.[5]They have proposed a solution in which, in order to recognize human posture, they segment moving human body from a video by applying visual surveillance intelligent platform (VSIP). Also in the work by Michael J. Jones et. al [6]. The proposed solution of human skin detection from and image is presented using various color models. For this purpose a threshold for skin color is defined. Elena Sanchez-Nielsen et. al. [7] uses a common method of hand gesture recognition that is through skin portion detection from an image. They chose HIS Hue, Saturation and Intensity color space to detect skin color.Another exceptional concept has been presented in the work of Jure Kovac et. $a l$.[8].The authors proposes to use two methods of skin color clustering, first they have used RGB color space and calculated a skin color threshold value, later they used $\mathrm{YCbCr}$ color space for the same purpose.M. Masud ur Rahman et. al. discussed in their work [9] have discussed the notion of figure effect and dress effect. The notion of figure is used to reduce the effect of change in figures of different humans and dress effect to enhance the result by reducing effect created by different dresses. Peter O'Donovan [10] states that for static gesture recognition a better approach is Restricted Boltzmann Machine. He says that, this technique is even better than other classification methods like Neural Nets and knearest neighbor rule. X. Zabulis et. al. [11] have reviewed multiple techniques related to recognition of hand gestures. The techniques include color and shape based hand detection, learning detectors from pixel values, 3D modeling, motion and tracking based detection and tracking based on templates and mean shift algorithm.

Antonio Chella et. al. in [12] have discussed methods for tracking humans in a video and recognition of his/her gesture. They used condensation algorithm and a modified eigenspace technique for posture recognition. Further Hao Jiang, et. al. in [13] uses linear programming technique for finding objects of interest. They compare the posture by searching for best matching region. Jie Yang et. al. proposes a system in [14]. This system, rather than using geometric features of human body it converts the gestures into sequential symbols. Jong-Seung Park et. al. in [15], presented a posture recognition method from single image. They suggest image segmentation into homogeneous regions, and thenthey segment curvedfeatures matching human limbs. l. Paniniet. al. [16] uses histogram projections to classify people's postures. Humberto Souto et. al. in [17] uses Artificial Neural Networks to gather information regarding human postures.

\section{Description of the System}

The system initiates with the acquisition of sequence of images from a web camera connected to the PC. Any standard web cam that supports resolution of $320 * 240$ (most commonly supported resolution) will suffice. The next step is to reduce or remove noise factor from the images for obtaining accurate results. Noise is reduced by performing preprocessing step. In the next phase background is removed and the human body is identified and hence segmented. To tackle this problem a powerful and efficient solution is provided which exploits the recognition capability of artificial neural nets. Making use of certain tool boxes and librarieswhich support neural network operation, the position of human body is extracted. After the images have been modified accurately to the specified requirementsthey are fed into the neural net for training. The result of the testing phase is the classification of posture into the 49 output classes. After the class of a posture is acquired it is transferred to XNA game studio to be converted into a replicated action mapped on a model made $\mathrm{n}$ blender and imported to visual studio 2008 .

$134 \quad$ Journal of Intelligent Computing Volume 9 Number 4 December 2018




\section{System Model}

The system model is described the diagram below (Figure 1), which shows the steps starting from the principal step of image acquisition to the final step of making the model move. These steps will further explained in detail.

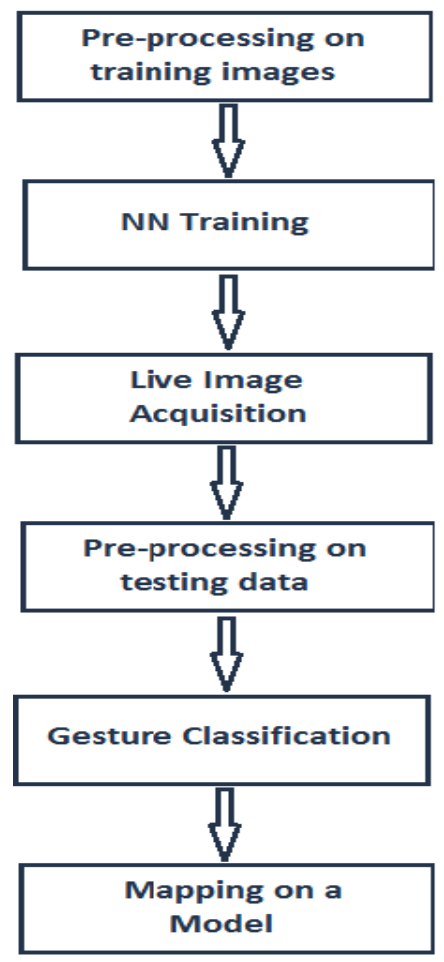

Figure 1. The System Model

\subsection{Image Acquisition}

Using the standard camera, images were taken which serve as the training data set. Each image had to undergo through preprocessing phase before going into the training phase. The system is designed to recognize 49 postures therefore a total of 49 classes are made with each class containing 40 images making a total of 1960 images. Images were acquired one by one from their respective classes for preprocessing.

\subsection{RGB to YCbCr}

Each image is converted from RGB to YCbCr. This conversion isperformed to detect skin color tones in each pixel of the image. In $\mathrm{YCbCr}$ ' $\mathrm{Y}$ ' is the luma component which means luminance and ' $\mathrm{Cb}$ ' and ' $\mathrm{Cr}$ ' are the blue and red components of the color space respectively. $\mathrm{YCbCr}$ is not an absolute color space, it is a way of encoding RGB information.

\subsection{Color filter (Based On human skin tone range)}

A sequence of elaborate skin filters are applied to the image and the human skin is extracted based on the human skin range exhibited by the following formula

$$
[r, c, v]=\operatorname{find}(C b>=77 \& C b<=127 \& C r>=133 \& C r<=173)
$$

Where $r, c$ and $v$ are the resultant components of the $\mathrm{YCbCr}$ conversion.

\subsection{Binarization}

Binarization means to convert an image into black and white form. ' 0 ' value in a pixel represents black color and ' 1 ' value represents white. The use of this filter was mandatory because it was desirable to convert the whole image including the background into black while the skin is kept white. 

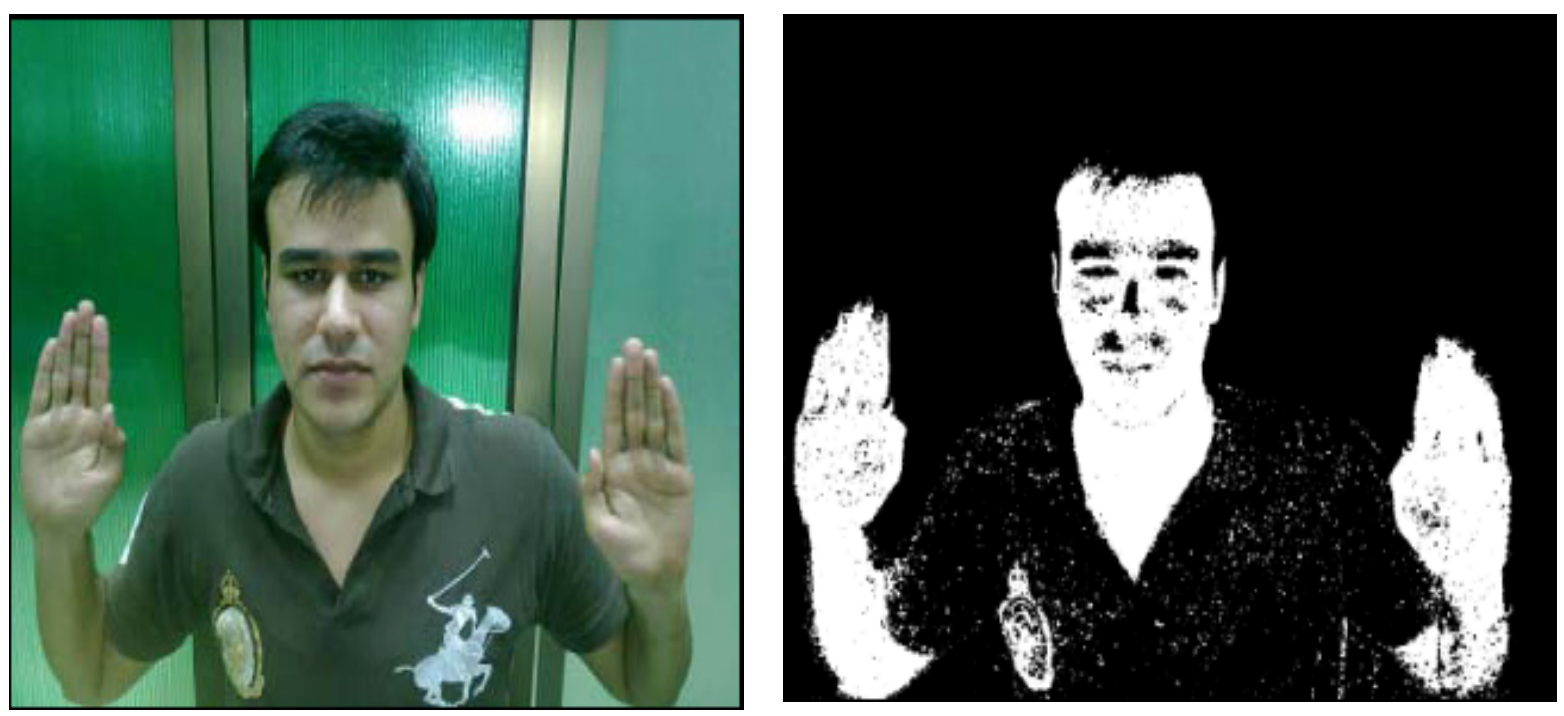

Figure 2. Shows Binarization of an Image

\subsection{Feature Extraction}

So far the image that is being processed has dimensions of $320 * 240$ which is small if we look at the sizes of everyday pictures we encounter. But for it to be fed into the neural network and to have run it through the intense preprocessing will lead to a lot of time spent on just the training phase. Moreover, if the number of pictures are in thousands (approximately 2000), it will not only be a huge resource consumer but also a computationally demanding job to go along with it. So clearly something needs to be done to downsize the dimensions of the image while keeping intact the semantic details of the image. For example an image has edges, vertical, horizontal and diagonal. When we reduce the size of an image to let's say, about $1 / 2$ times, such information tends to get lost in the compression, especially if the size of the image is already small. Therefore we need a solution that not only reduces the dimensions of the image but also preserves, or better yet, enhances the detail found in the image. This is where the Discrete Wavelet Transformation (DWT) steps in. DWT is the type of transformation in which all the component wavelets of a signal are discretely sampled. A Discrete Fourier Transform (DFT) only extracts the frequency components whereas a DWT also extracts the temporal information of the signals. One of the most popular and efficient type of wavelet transform is the Haar wavelet. The haar wavelet is based on the Haar function $\psi_{n, k}$ defined on real line $\mathbb{R}$ given as

$$
\psi_{n, k}(t)=2^{\frac{n}{2}} \psi\left(2^{n} t-k\right), t \in \mathbb{R}
$$

Where $n$ and $k$ are positive integers. A useful property of Haar function is that it is pairwise orthogonal

$$
\int \psi_{n_{1} k_{1}}(t) \psi_{n_{2} k_{2}}(t) d t=\delta_{n_{1} n_{2}} \delta_{k_{1} k_{2}}
$$

Where

$$
\delta_{i, j}=\left\{\begin{array}{l}
1 \text { if } i=j \\
0 \text { if } i \neq j
\end{array}\right.
$$

Based on the Haar wavelet function a Haar matrix [18] is derived given as

$$
H_{4}=\frac{1}{\sqrt{4}}\left[\begin{array}{cccc}
1 & 1 & 1 & 1 \\
1 & 1 & -1 & -1 \\
\sqrt{2} & -\sqrt{2} & 0 & 0 \\
0 & 0 & \sqrt{2} & -\sqrt{2}
\end{array}\right]
$$

Following is the result of performing Haar wavelet transform on a frame

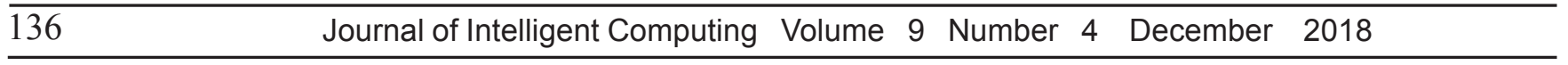




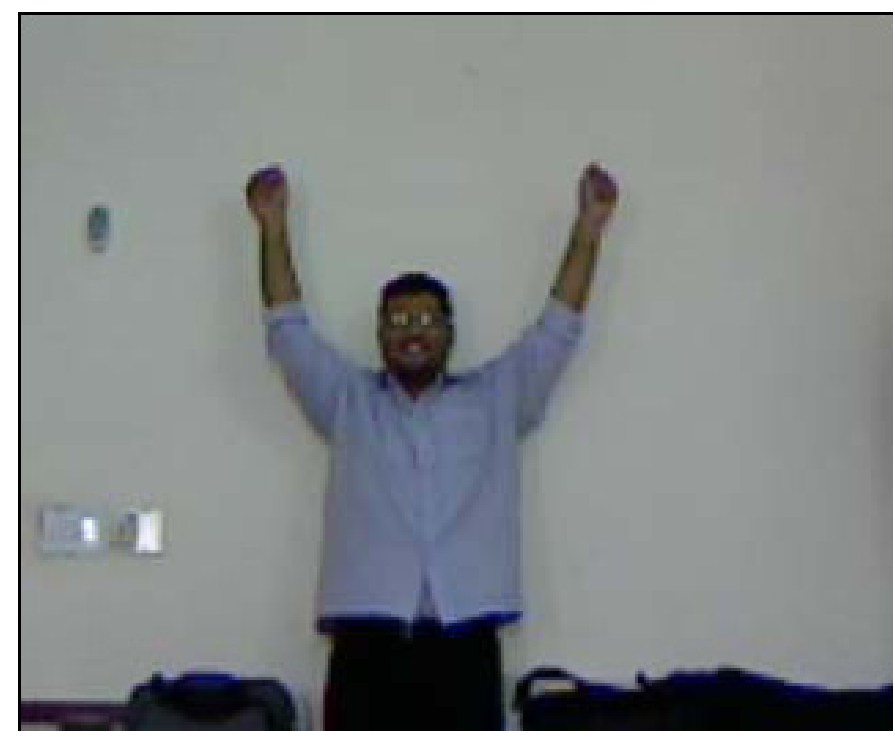

Figure 3. Shows an arbitrary Input Image

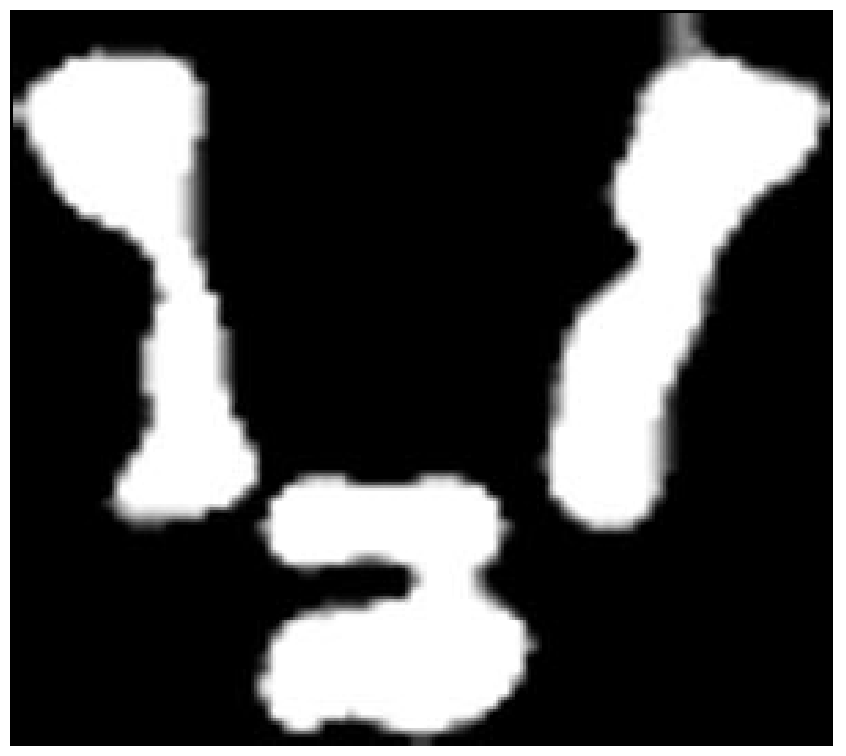

Figure 4. Shows the Image of Figure 3 After Application of Skin Filter

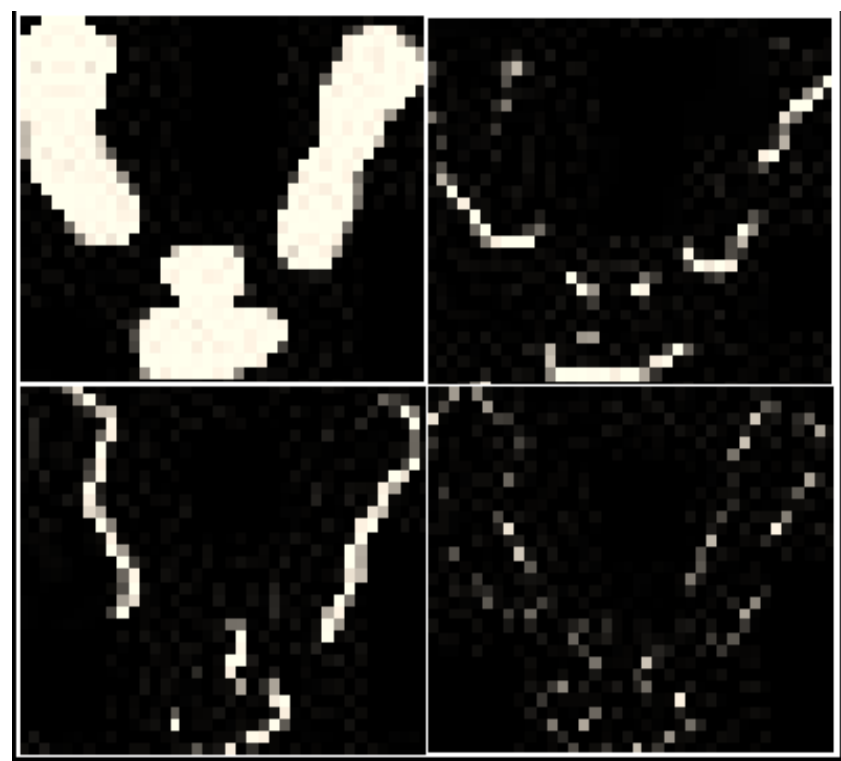

Figure 5. Shows the Image in Figure 4 after Application Haar Wavelet Transform

\section{Artificial Neural Network (ANN)}

An ANN is a mathematical or computational model that is inspired by the structure and functional aspects of biological neural networks. A neural network consists of an interconnected group of artificial neurons, and it processes information using a connectionist approach to computation. In most cases an Artificial Neural Network is an adaptive system that changes its structure based on external or internal information that flows through the network during the learning phase.For the proposed problem a pattern recognition network is developed, using back propagation algorithm. Back Propagation is the technique in which error gradient is calculated by the output layer. Based on this error the weights of the neurons are adjusted. This adjustment is propagated back to the previous layer, in order to calculate its errors.Other key factors that play a crucial role in development of a neural network are selection and number of the input images, number of hidden neuron, training functions, number of epochs, learning rate and error gradient. 
The pre-processed Images, act as input to the network. Hidden neurons are initially assigned weights randomly. The activation function used is Gradient Descent with adaptive learning back propagation. Gradient Descent with adaptive learning back propagation is a network training function that updates weight and bias values according to gradient descent with adaptive learning rate.

Gradient Descent with adaptive learning back propagationcan train any network as long as its weight, net input, and transfer functions have derivative functions. Back propagation is used to calculate derivatives of performance termed as "dperf" with respect to the weight and bias variables $X$. Each variable is adjusted according to the gradient descent. $L r$ is the learning rate, the change in $X$ is given as:

$$
d X=l r * \text { dpref } / d X
$$

At each epoch, if performance decreases toward the goal, then the learning rate is increased. If performance increases by more than the factor, then learning rate is adjusted by the factor and the changeis not made. Here are few of the equations used in the Gradient Descent with adaptive learning back propagation.

Total error in the network was calculated using following equation.

$$
\mathrm{E}=\frac{1}{2} \sum\left(t_{k}-a_{k}\right)^{2}
$$

The equation used to adjust the network weights is as follows

$$
\Delta \mathcal{W} \propto-\eta \frac{\partial E}{\partial \mathcal{W}}
$$

where $\eta$ represents the step size and is called the learning rate.

The derivative of the error with respect to the activation is calculated by the equation below.

$$
\frac{\partial \mathrm{E}}{\partial a_{k}}=\frac{\partial\left(\frac{1}{2}\left(t_{k}-a_{k}\right)^{2}\right.}{\partial a_{k}}=-\left(t_{k}-a_{k}\right)
$$

The weight change rule for hidden to output weights is

$$
\omega_{k j}=\eta \delta_{k} \alpha_{i}
$$

where the term $\delta_{k}$ represents the product of the error with the derivative of the activation function [19-23].

Weight change rule used for an input to hidden weight is

$$
\Delta \omega_{k j}=\eta \delta_{j} \alpha_{i}
$$

Several combinations of parameters were probed and modified in order to improve the results. Initially the network input with 49 gestures and 20 samples for each was tried, later it was increased to 40 samples for each gesture. Final training parameters and their result is provided below.

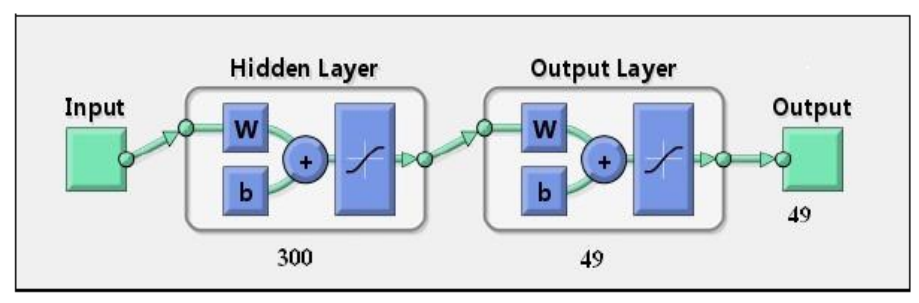

Figure 6. Shows the Training Process

\begin{tabular}{llllll}
\hline $138 \quad$ Journal of Intelligent Computing Volume & 9 & Number & 4 & December & 2018 \\
\hline
\end{tabular}


hiddenNeurons $=300$;

trainFunction = 'traingda';

totalEpochs $=1500$

learningRate $=0.0019$;

goal $=1 \mathrm{e}-5$;

$\operatorname{maxFail}=5$;

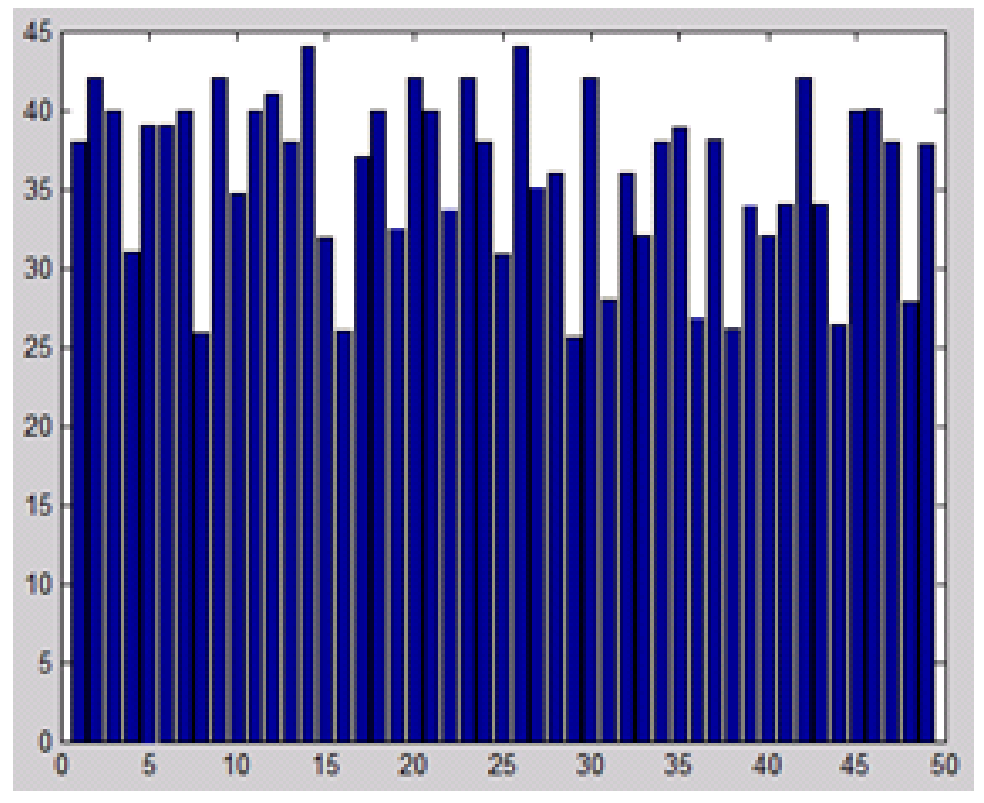

Figure 7. Shows the Accuracy in Recognition for each Class

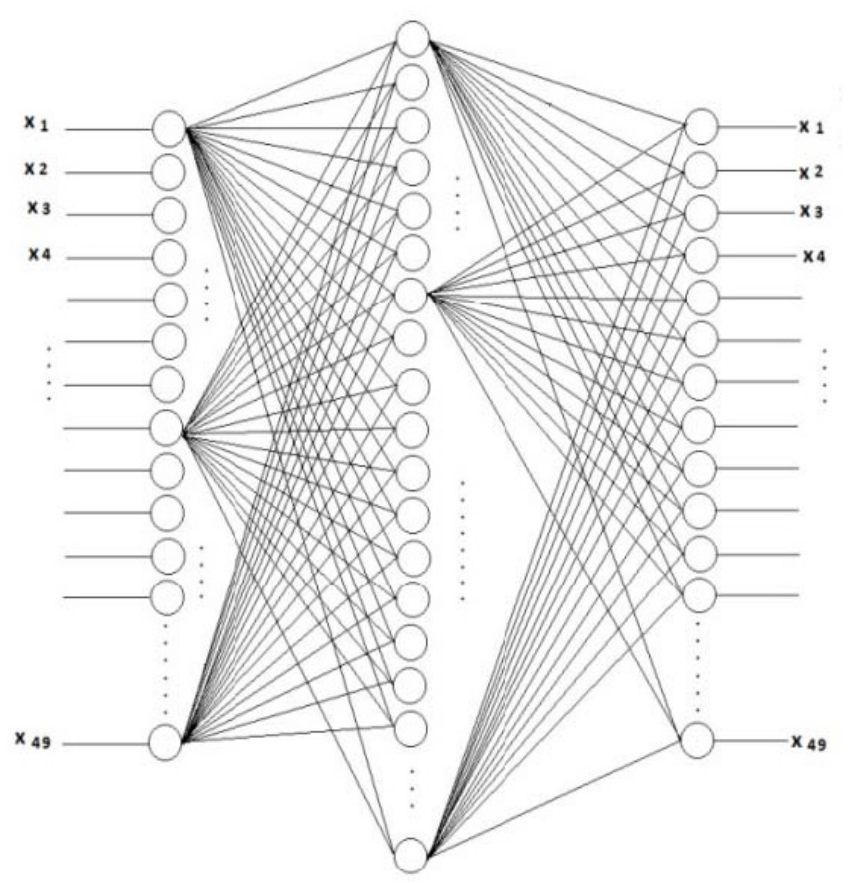

Figure 8. Shows the Connections of the Neural Network 


\section{Testing}

The network is trained with 49 gestures to recognize using approximately 2000 images and state of the art algorithms. The trained neural net has refined weights that form the backbone of input classification. An input is basically presented to the neural net minus the back propagation algorithm. After the multiplication of the input with the weights defined by the activation function the result is acquired.

The neural net is clamped inputs from images that have already been preprocessed. The first target therefore is acquiring the images.

\subsection{Image Acquisition}

Images are acquired at regular intervals using a standard web cam.

\subsection{Live Pre processing}

The image is binarized and convolved through the skin filter, this leaves the skin portion of the image as white while rest of the image is black. Later a noise reduction algorithm selects blobs (erroneously selected as human skin on the basis of matching RGB values) and then eliminates them because they do not fall in the dimensional range of human head and arm. The denoised image is transformed into a feature vector using Discrete Wavelet Transform with Haar like features.

\subsection{Live Neural Net Testing}

The feature vector is thenclamped to the neural net for testing. This classifies the image to one of the 49 classes of postures. The result is a 1 dimensional array containing the detected class. A correlation of this output array with the input features input array is used to establish the accuracy of the system.

\section{Model Making:}

In making a model, the major concern was to find a 3D designing tool that offered compatibility with IDEs so that the model that was created could be manipulated producing a three dimensional effect. For thispurpose Blender was chosen as the tool for model making. The model created had bones with hierarchy of parent child relationship and was skinned/rigged for the purpose of replication of human motion replication.

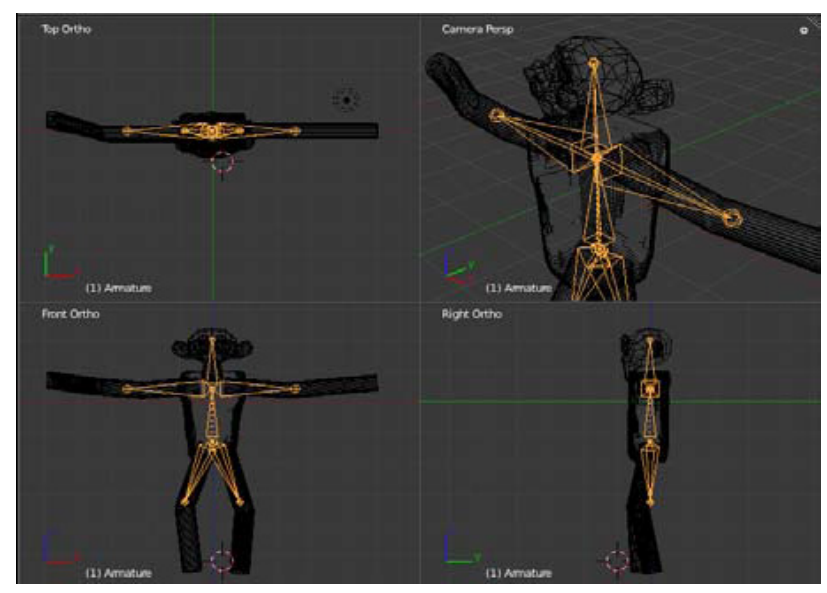

Figure 9. Model Designed in XNA

\section{Using XNA}

Together XNA Game Studio 3.1 and Microsoft Visual C\# 2008 Express provide an intuitive development environment that supports the creation of computer games. They provide everything needed to create, test, run, and debug computer games on a Windows computer. XNA Game Studio 3.1 supplies access to an enormous collection of pre-written program code, which provides a simplified game development platform.

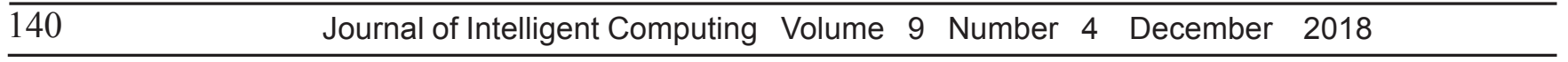


Using properties of the models and basic transformations like rotation, scale and translation the bones of the model are manipulated and moved as desired.

The resultant one dimensional array of the ANN live output is used to acquire the detected class of the posture and hence the bones of the model are animated, such that its motion mimics the action performed by the user.

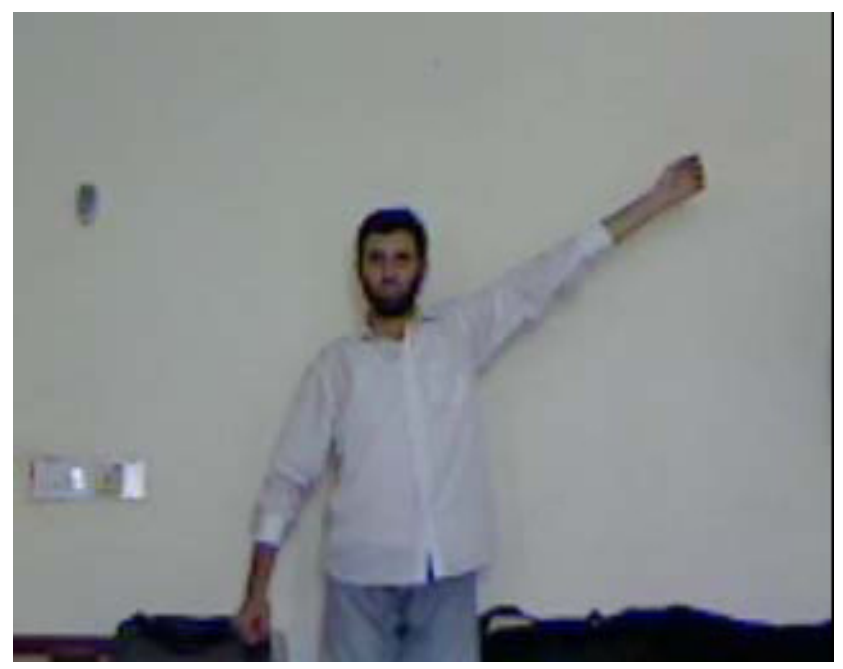

Figure 10. Live Camera Feed

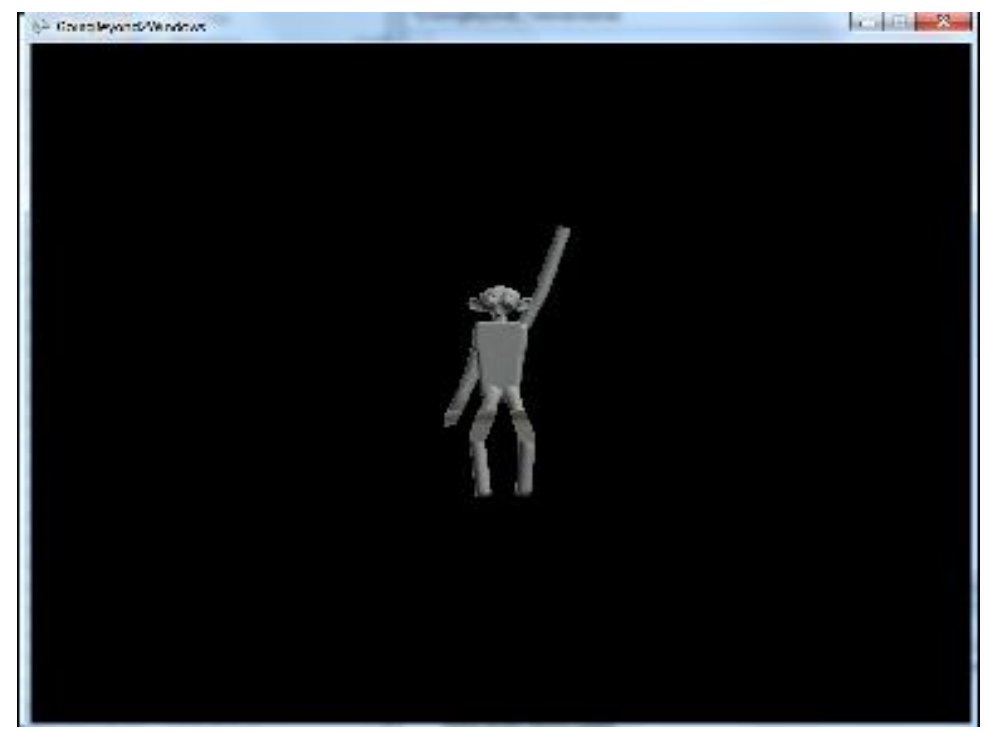

Figure 11. Mimicking Action of the Model

\section{Conclusion}

The system is recognized the human activity and was satisfactorily able to imitate the activity. It requires only a web cam. As opposed to an expensive console that otherwise would be quite expensive. Almost everyone has a computer lying around. And the domain of the computer (PC) is way larger than the domain of the consoles. One can say that the console titles are a product of one of the area of the domain the computer field is the master of.

It provides a substantial base to what future systems of interaction may look like. The system even though based for a specific environment currently is applicable to almost any human activity as long as there is skin exposed. The recognition can be done 


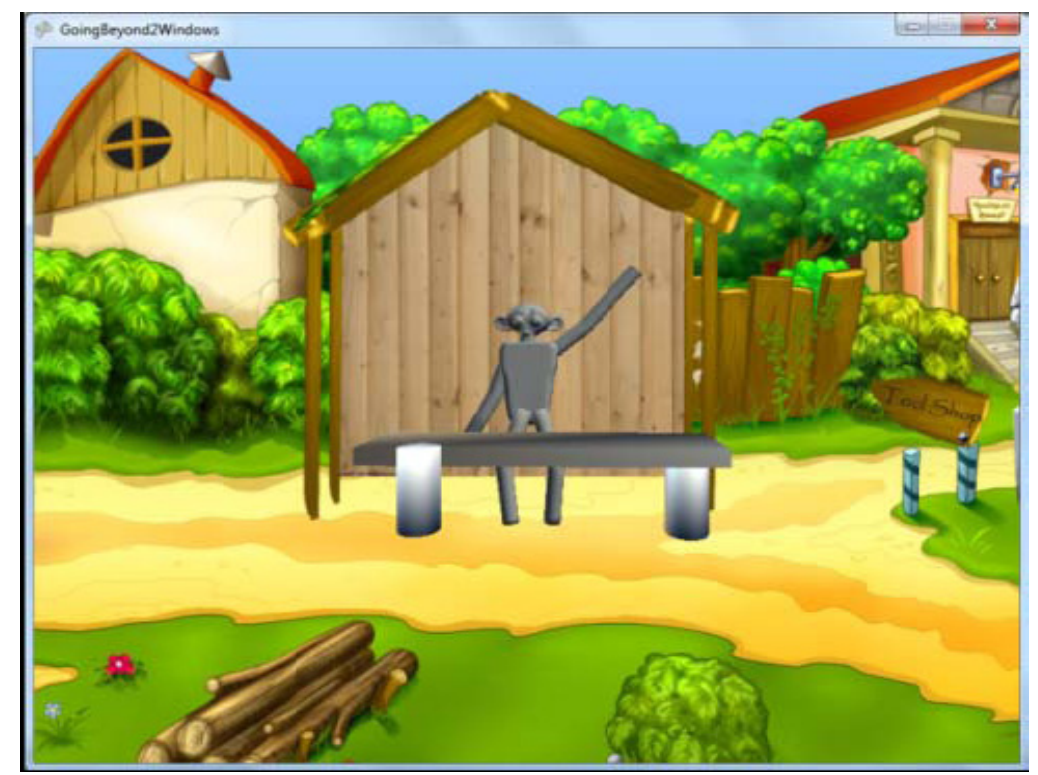

Figure 12. The Action has been mapped onto a Game Scenario

by only replacing the current data set with the dataset of the new activity.

The action recognition can be applied in various ways besides the one described in this article. For instance in a medical field they could be used to set off an alarm in case of detection of a collapsing patient. A child could be taught to choose the correct alphabet based on the position of the screen he points at. The gist of the conclusion is that with this system as a base the possibilities of its areas of implementation now are endless.

\section{References}

[1] Kaaniche, M. B., Bremond, F. (2010, June).Gesture recognition by learning local motion signatures. In: Computer Vision and Pattern Recognition (CVPR), 2010 IEEE Conference on (2745-2752). IEEE.Chicago.

[2] Srinivasan, K., Porkumaran, K., Sainarayanan, G. (2009). Intelligent human body tracking, modeling, and activity analysis of video surveillance system: A survey. Int. J. of Convergence in Engineering, Technology and Science, 1, 1-8.

[3] Al-Jarrah, O., Halawani, A. (2001). Recognition of gestures in Arabic sign language using neuro-fuzzy systems. Artificial Intelligence, 133 (1) 117-138.

[4] Wu, Y., Liu, Q., Huang, T. S. (2000). An adaptive self-organizing color segmentation algorithm with application to robust realtime human hand localization. In: Proceedings of Asian Conference on Computer Vision (1106- 1111).

[5] Boulay, B., Bremond, F., Thonnat, M. (2003). Human posture recognition in video sequence. In: IEEE International Workshop on VS-PETS, Visual Surveillance and Performance Evaluation of Tracking and Surveillance.

[6] Jones, M. J., Rehg, J. M. (2002). Statistical color models with application to skin detection. International Journal of Computer Vision, 46 (1) 81-96.

[7] Sánchez-Nielsen, E., Antón-Canalís, L., Hernández-Tejera, M. (2004). Hand gesture recognition for human-machine interaction.

[8] Kovac, J., Peer, P., \&Solina, F. (2003). Human skin color clustering for face detection (2, 144-148).IEEE.

[9] Rahman, M. M., Ishikawa, S. (2005). Human motion recognition using an eigenspace. Pattern Recognition Letters, 26 (6) 687697.

[10] O’Donovan, P. Static Gesture Recognition with Restricted Boltzmann Machines.

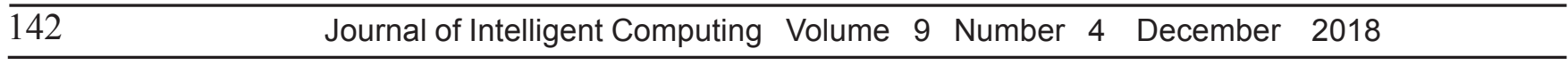


[11] Zabulis, X., Baltzakis, H., Argyros, A. (2009). Vision-based hand gesture recognition for human-computer interaction.The Universal Access Handbook, 34-1.

[12] Chella, A., Dindo, H., Infantino, I. (2005, November). A system for simultaneous people tracking and posture recognition in the context of human-computer interaction, In: Computer as a Tool, 2005.EUROCON 2005. The International Conference on $(2,991-$ 994).IEEE.

[13] Jiang, H., Drew, M. S., Li, Z. N. (2006). Successive convex matching for action detection. In: Computer Vision and Pattern Recognition, 2006, IEEE Computer Society Conference on (2, 1646-1653). IEEE.

[14 ]Yang, J., Xu, Y. (1994). Hidden markov model for gesture recognition (No.CMU-RI-TR- 94-10).CARNEGIE-MELLON UNIV PITTSBURGH PAROBOTICS INST.

[15] Park, J., Oh, H., Chang, D., Lee, E. (1999, December). Human posture recognition using curved segments for image retrieval. In Electronic Imaging (p 2-11). International Society for Optics and Photonics.

[16] Panini, L., Cucchiara, R. (2003, September). A machine learning approach for human posture detection in domotics applications, In: Image Analysis and Processing, 2003. Proceedings. $12^{\text {th }}$ International Conference on (103-108). IEEE.

[17] Braun, H., Souto, H., Jacques, J. C. S., Dihl, L. L., Braun, A., Musse, S. R., Keshet, R. (2011, November). Making them alive. In: Games and Digital Entertainment (SBGAMES), 2011 Brazilian Symposium on (182-188). IEEE.

[18] Vidakovic, B. (2009). Statistical modeling by wavelets (503). John Wiley \& Sons.

[19] Khan, YD., Ahmad, F., Anwar, MW. (2016). A neuro-cognitive approach for iris recognition using back propagation, World Applied Sciences Journal 16 (5) 678-685.

[20] Butt, AH., Khan, SA., Jamil, H., Rasool, N., Khan, YD. (2016). A prediction model for membrane proteins using moments based features, BioMed Research International.

[21] Khan, Y D., Khan, S A. A Survey on use of Neuro-Cognitive and Probabilistic Paradigms in Pattern Recognition, Res. J. Recent Sci.2 (4) 74-79

[22] Ehsan, A., Mahmood, K., Khan, YD., Khan, SA., KC Chou, A Novel Modeling in Mathematical Biology for Classification of Signal Peptides, Scientific reports 8 (1) 1039

[23] MA Akmal, N Rasool, YD Khan, Prediction of N-linked glycosylation sites using position relative features and statistical moments, PloS one 12 (8) e0181966 